\title{
An a posteriori Fourier regularization method for identifying the unknown source of the space-fractional diffusion equation
}

\author{
Xiao-Xiao Li, Jin Li Lei and Fan Yang*
}

\section{*Correspondence:}

yfggd114@163.com

School of Science, Lanzhou

University of Technology, Lanzhou,

Gansu 730050, People's Republic of China

\begin{abstract}
In this paper, we identify the unknown source which depends only on spatial variable for a fractional diffusion equation using the Fourier method. Not alike the previous literature, we propose to choose the regularization parameter by an a posteriori rule, with which we can obtain error estimate of Hölder type between the exact solution and the regularized approximation. Numerical simulations show that the proposed scheme is effective and stable.
\end{abstract}

MSC: 35R30; 47A52; 65M30; 65M32

Keywords: unknown source; space-fractional diffusion equation; Fourier regularization method; a posteriori parameter choice; error estimate

\section{Introduction}

Nowadays, the study of fractional differential equations receives a lot of attention. The interest in fractional calculus and fractional differential equations has rapidly increased among engineers and scientists due to their vast potential of applications, including physical, chemical, mechanical engineering, signal processing and systems identification, biology systems, control theory, finance etc. In fact, it can be noticed that one of the most successful and concrete applications of fractional calculus and fractional differential equations is to effectively characterize the anomalous diffusion. It is well known that the ordinary diffusion process is intimately related to the validity of the central limit theorem, which is characterized by the linear dependence of the mean square displacement $\left\langle x^{2}(t)\right\rangle \sim \kappa t$ with the diffusion coefficient $\kappa$. However, some diffusion processes, especially in various complex systems, no longer follow Gaussian behavior. This phenomenon is named anomalous diffusion which is described by the nonlinear growth of the mean square displacement $x(t)$ of a diffusion particle over time $t:\left\langle x^{2}(t)\right\rangle \sim \kappa_{\alpha} t^{\alpha}$, where $\kappa_{\alpha}$ is the diffusion coefficient, and $\alpha$ is the anomalous diffusion exponent. For different $\alpha$, the anomalous diffusion is classified into subdiffusion $(0<\alpha<1)$, normal diffusion $(\alpha=1)$, superdiffusion $(\alpha>1)$, and ballistic diffusion $(\alpha=2)[1,2]$. And the Fick's law is inevitable to be modified in order to precisely describe the anomalous diffusion behavior [3].

Much progress has been made for numerically solving space fractional partial differential equations [3-9]. Here, instead of further pursuing research in this direction, we discuss the space fractional inverse diffusion equation, i.e., to determine an unknown source,

○2014 Li et al.; licensee Springer. This is an Open Access article distributed under the terms of the Creative Commons Attribution License (http://creativecommons.org/licenses/by/2.0), which permits unrestricted use, distribution, and reproduction in any medium, provided the original work is properly cited. 
which depends only on the spatial variable, in the one-dimensional space fractional diffusion equation. Determination of an unknown source is obtaining the information about a physical object or system by observed datum, and it is one of the most important and well-studied problems in many branches of engineering sciences, e.g., heat conduction, crack identification, electromagnetic theory, geophysical prospecting and pollutant detection. For the heat source identification, there have been a large number of research results for different forms of heat source [10-17]. To the authors' knowledge, there were few papers for identifying an unknown source for a fractional diffusion equation by the regularization method. In [18], the authors proved the uniqueness of the identification of the unknown source dependent only on spatial variable for the fractional diffusion equation in a bound domain. In [19], using the coupled method, the authors identified the unknown source for the spatial fractional diffusion equations. In [20], the authors identified the unknown source for the time fractional diffusion equation using the mollification method. In [21], the authors identified the unknown source dependent only on time variable in a time-fractional diffusion equation using the boundary element method. In [22], the authors identified the unknown source dependent only on spatial variable for the timefractional diffusion equation using the Tikhonov regularization method and the simplified Tikhonov regularization method, respectively. In [23], the authors identified the unknown source dependent only on spatial variable for the time-fractional diffusion equation using the truncation regularization method.

In this paper, we consider the following inverse source problems of determining the unknown source term $f(x)$, in the following a Riesz-Feller space-fractional equation:

$$
\begin{cases}u_{t}(x, t)-{ }_{x} D_{\theta}^{\alpha} u(x, t)=f(x), & x \in \mathbb{R}, 0<t<1, \\ u(x, 0)=0, & x \in \mathbb{R}, \\ u(x, 1)=g(x), & x \in \mathbb{R},\end{cases}
$$

where the space-fractional derivative ${ }_{x} D_{\theta}^{\alpha}$ is the Riesz-Feller fractional derivative of order $\alpha(0<\alpha<2)$ and skewness $\theta(|\theta| \leq \min \{\alpha, 2-\alpha\}, \theta \neq \pm 1)$ which is defined by the Fourier transform in [24], i.e., [25]

$$
\mathcal{F}\left\{{ }_{x} D_{\theta}^{\alpha} f(x) ; \xi\right\}=-\psi_{\alpha}^{\theta}(\xi) \hat{f}(\xi)
$$

where

$$
\psi_{\alpha}^{\theta}(\xi)=|\xi|^{\alpha} e^{i(\operatorname{sign}(\xi)) \frac{\theta \pi}{2}}
$$

Moreover, from [24], the Riesz-Feller fractional derivative can be written as

$$
\begin{aligned}
{ }_{x} D_{\theta}^{\alpha} f(x)= & \frac{\Gamma(1+\alpha)}{\pi}\left\{\sin \frac{(\alpha+\theta) \pi}{2} \int_{0}^{\infty} \frac{f(x+\xi)-f(x)}{\xi^{1+\alpha}} d \xi\right. \\
& \left.+\sin \frac{(\alpha-\theta) \pi}{2} \int_{0}^{\infty} \frac{f(x+\xi)-f(x)}{\xi^{1+\alpha}} d \xi\right\}, \quad 0<\alpha<2, \\
{ }_{x} D_{0}^{2} f(x)= & \frac{d^{2} f(x)}{d x^{2}}, \quad \alpha=2,
\end{aligned}
$$


where $\Gamma(\cdot)$ is a gamma function. However, for the convenience of numerical calculation, the Riesz-Feller fractional derivative ${ }_{x} D_{\theta}^{\alpha}$ can also be defined as follows (see [26]):

$$
\begin{aligned}
{ }_{x} D_{\theta}^{\alpha} f(x)= & -\frac{1}{\Gamma(1-\alpha) \sin (\alpha \pi)}\left\{\sin \frac{(\alpha-\theta) \pi}{2} \frac{d}{d x} \int_{-\infty}^{x} \frac{f(\xi)}{(x-\xi)^{\alpha}} d \xi\right. \\
& \left.+\sin \frac{(\alpha+\theta) \pi}{2} \frac{d}{d x} \int_{x}^{\infty} \frac{f(\xi)}{(\xi-x)^{\alpha}} d \xi\right\}, \quad 0<\alpha<1, \\
{ }_{x} D_{\theta}^{1} f(x)= & -\frac{1}{\pi} \cos \frac{\theta \pi}{2} \frac{d}{d x} \int_{-\infty}^{\infty} \frac{f(\xi)}{x-\xi} d \xi+\sin \frac{\theta \pi}{2} \frac{d f(x)}{d x}, \quad \alpha=1, \\
{ }_{x} D_{\theta}^{\alpha} f(x)= & -\frac{1}{\Gamma(2-\alpha) \sin (\alpha \pi)}\left\{\sin \frac{(\alpha-\theta) \pi}{2} \frac{d^{2}}{d x^{2}} \int_{-\infty}^{x} \frac{f(\xi)}{(x-\xi)^{\alpha-1}} d \xi\right. \\
& \left.+\sin \frac{(\alpha+\theta) \pi}{2} \frac{d^{2}}{d x^{2}} \int_{x}^{\infty} \frac{f(\xi)}{(\xi-x)^{\alpha-1}} d \xi\right\}, \quad 1<\alpha<2, \\
{ }_{x} D_{0}^{2} f(x)= & \frac{d^{2} f(x)}{d x^{2}}, \quad \alpha=2 .
\end{aligned}
$$

$f(x)$ denotes the source term. Our purpose is to identify $f(x)$ from the additional data $u(x, 1)=g(x)$. Since the data $g(x)$ is based on (physical) observation, there must exist measurement errors, and we assume the measured data $g_{\delta}(x) \in L^{2}(\mathbb{R})$, which satisfies

$$
\left\|g-g_{\delta}\right\| \leq \delta
$$

where $\|\cdot\|$ denotes $L^{2}$-norm and the constant $\delta>0$ is a noise level.

The problem is ill-posed in the sense of Hadamard, i.e., small changes in the measured data can blow up the solution. The ill-posedness can be seen by solving the problem in the frequency domain. In order to analyze problem $(1.1)$ in $L^{2}(\mathbb{R})$, we define

$$
\hat{f}(\xi):=\frac{1}{\sqrt{2 \pi}} \int_{-\infty}^{\infty} e^{-i \xi x} f(x) d x,
$$

which is the Fourier transform of the function $f(x)$.

Using the Fourier transform, we obtain the solution of (1.1) as follows:

$$
\hat{f}(\xi)=\frac{\psi_{\alpha}^{\theta}(\xi)}{1-e^{-\psi_{\alpha}^{\theta}(\xi)}} \hat{g}(\xi)
$$

or equivalently,

$$
f(x)=\frac{1}{\sqrt{2 \pi}} \int_{-\infty}^{\infty} e^{i \xi x} \frac{\psi_{\alpha}^{\theta}(\xi)}{1-e^{-\psi_{\alpha}^{\theta}(\xi)}} \hat{g}(\xi) d \xi
$$

Note that $\psi_{\alpha}^{\theta}(\xi)(|\theta| \leq \min \{\alpha, 2-\alpha\}, \theta \neq \pm 1)$ has a positive real part $|\xi|^{\alpha}$, the small error in the high-frequency components will be amplified by the factor $|\xi|^{\alpha}$ as $|\xi| \rightarrow \infty$. The small disturbance for the data $g(x)$ will be amplified infinitely by this factor and lead to the integral (1.7) blow-up. So identifying the unknown source $f(x)$ from the measured data $g_{\delta}(x)$ is severely ill-posed. Therefore, when we consider our problem in $L^{2}(\mathbb{R})$, the exact data function $\hat{g}(\xi)$ must decay fast. However, the measured data function $g_{\delta}(x)$, which is 
merely in $L^{2}(\mathbb{R})$, does not posses such a decay property in general. Thus if we try to obtain the unknown source $f(x)$, high frequency components in the error are magnified and can destroy the solution. It is impossible to solve problem (1.1) by using classical methods. In the following section, we will use the Fourier regularization method to deal with the ill-posed problem. Before doing that, we impose an a priori bound on the input data, i.e.,

$$
\|f(\cdot)\|_{H^{p}} \leq E, \quad p>0,
$$

where $E>0$ is a constant, $\|\cdot\|_{H^{p}}$ denotes the norm in the Sobolev space $H^{p}(\mathbb{R})$ defined by

$$
\|f(\cdot)\|_{H^{p}}:=\left(\int_{-\infty}^{\infty}|\hat{f}(\xi)|^{2}\left(1+\xi^{2}\right)^{p} d \xi\right)^{\frac{1}{2}}
$$

In [27], the authors used the Fourier method to identify the unknown source which depends only on the spatial variable for the space-fractional diffusion equation, but the regularization parameter is an a priori choice rule. Generally speaking, there is a defect in any a priori methods; i.e., the a priori choice of the regularization parameter depends obviously on the a priori bound $E$ of the unknown solution. But the a priori bound $E$ cannot be known exactly in practice, and working with a wrong constant $E$ may lead to the bad regularization solution. In the present paper, an a posteriori choice of the regularization parameter will be given. To the authors' knowledge, there are few papers for choosing the regularization parameter by the a posteriori rule for this problem.

The Fourier regularization method has been studied for solving various types of inverse problems. Eldén et al. [28] used the truncation method to analyze and compute a onedimensional inverse heat conduction problem. Xiong et al. [29] used it to consider the surface heat flux for the sideways heat equation. Fu et al. [30] used it to solve the backward heat conduction problem. Qian et al. [31] used it to consider the numerical differentiation. Regińska and Regiński [32] applied it to a Cauchy problem for the Helmholtz equation. Dou et al. [33] used it to identify the unknown heat source dependent only on spatial variable. Yang and $\mathrm{Fu}$ [34] used it to identify the unknown heat source dependent only on time variable. But in these papers, the regularization parameters were an a priori choice rule. In this paper, we will give the a posteriori choice rule for identifying the unknown source in the fractional diffusion equation.

The outline of the paper is as follows. Section 2 gives some auxiliary results, the Fourier regularization method and an a posteriori parameter choice rule. In Section 3, some numerical examples are proposed to show the effectiveness of this method. Section 4 puts an end to this paper with a brief conclusion.

\section{An a posteriori regularization parameter choice rule for the Fourier method and convergence estimate}

It is obvious that the ill-posedness of problem (1.1) is caused by disturbance of high frequencies. A natural way to stabilize problem (1.1) is to eliminate all high frequencies from the solution $f(x)$. We define a regularization approximation solution of problem (1.1) for noisy data $g_{\delta}(x)$ as follows:

$$
f_{\delta, \xi \max }(x):=\frac{1}{\sqrt{2 \pi}} \int_{-\infty}^{\infty} e^{i \xi x} \frac{\psi_{\alpha}^{\theta}(\xi)}{1-e^{-\psi_{\alpha}^{\theta}(\xi)}} \hat{g}_{\delta}(\xi) \chi_{\max } d \xi,
$$


which is called the Fourier truncation regularized solution of problem (1.1), where $\chi_{\max }$ is the characteristic function of the interval $\left[-\xi_{\max }, \xi_{\max }\right]$, i.e.,

$$
\chi_{\max }(\xi)= \begin{cases}1, & |\xi| \leq \xi_{\max } \\ 0, & |\xi|>\xi_{\max }\end{cases}
$$

and $\xi_{\max }$ is a constant which will be selected appropriately as regularization parameter. We consider an a posteriori regularization parameter choice by the discrepancy principle. Choose the regularization parameter $\xi_{\max }$ as the solution of the equation

$$
\left\|\left(1-\chi_{\max }(\xi)\right) \hat{g}_{\delta}(\xi)\right\|=\tau \delta, \quad \tau>1 \text { is a constant, }
$$

where $\chi_{\max }(\xi)$ is defined by (2.2). To establish the existence and uniqueness of the solution for equation (2.3), we need the following lemma and remark.

Lemma 2.1 Let $\rho\left(\xi_{\max }\right):=\left\|\left(1-\chi_{\max }(\xi)\right) \hat{g}_{\delta}(\xi)\right\|$, then, for $\delta>0$, the following hold:

(a) $\rho\left(\xi_{\max }\right)$ is a continuous function;

(b) $\lim _{\xi_{\max } \rightarrow \infty} \rho\left(\xi_{\max }\right)=0$;

(c) $\lim _{\xi_{\max } \rightarrow 0} \rho\left(\xi_{\max }\right)=\left\|\hat{g}_{\delta}\right\|$;

(d) $\rho\left(\xi_{\max }\right)$ is a strictly decreasing function.

The proof is very easy and we omit it here.

Remark 2.2 To establish the existence and uniqueness of the solution for equation (2.3), we always suppose $0<\delta<\left\|g_{\delta}\right\|$.

To establish the error estimate for the $a$ posteriori choice rule of the regularization parameter, we need the following lemmas.

Lemma 2.3 If $x>1$, the following inequality holds:

$$
\frac{1}{1-e^{-x}}<2
$$

Lemma 2.4 If $\xi \in \mathbb{R}$, the following inequality holds:

$$
\left|\frac{\psi_{\alpha}^{\theta}(\xi)}{1-e^{-\psi_{\alpha}^{\theta}(\xi)}}\right| \leq \frac{|\xi|^{\alpha}}{1-e^{-|\xi|^{\alpha} \cos \left(\frac{\theta \pi}{2}\right)}}
$$

Proof With the fact $\left|e^{z}\right|=e^{\operatorname{Re}(z)}$, we obtain

$$
\left|\frac{\psi_{\alpha}^{\theta}(\xi)}{1-e^{-\psi_{\alpha}^{\theta}(\xi)}}\right| \leq \frac{\left|\psi_{\alpha}^{\theta}(\xi)\right|}{|1-| e^{-\psi_{\alpha}^{\theta}(\xi)}||}=\frac{|\xi|^{\alpha}}{1-e^{-\operatorname{Re}\left(\psi_{\alpha}^{\theta}(\xi)\right)}}=\frac{|\xi|^{\alpha}}{1-e^{-|\xi|^{\alpha} \cos \left(\frac{\theta \pi}{2}\right)}} .
$$

Lemma 2.5 If $\xi_{\max }$ is the solution of Eq. (2.3), then the following inequality holds:

$$
\left|\xi_{\max }\right| \leq\left(\frac{2 E}{(\tau-1) \delta}\right)^{\frac{1}{p+\alpha}}
$$


Proof Due to (1.8), we obtain

$$
\begin{aligned}
\| & \left(1-\chi_{\max }(\xi)\right) \hat{g}(\xi) \| \\
& =\left(\int_{|\xi| \geq \xi_{\max }}|\hat{g}(\xi)|^{2} d \xi\right)^{\frac{1}{2}} \\
& =\left(\int_{|\xi| \geq \xi_{\max }}\left|\frac{\psi_{\alpha}^{\theta}(\xi)}{1-e^{-\psi \psi_{\alpha}^{\theta}(\xi)}} \hat{g}(\xi)\right|^{2}\left(1+\xi^{2}\right)^{p}\left|\frac{\psi_{\alpha}^{\theta}(\xi)}{1-e^{-\psi_{\alpha}^{\theta}(\xi)}}\right|^{-2}\left(1+\xi^{2}\right)^{-p} d \xi\right)^{\frac{1}{2}} \\
& \leq \sup _{|\xi| \geq \xi_{\max }}|| \frac{1-e^{-\psi_{\alpha}^{\theta}(\xi)}}{\psi_{\alpha}^{\theta}(\xi)}\left|\left(1+\xi^{2}\right)^{-\frac{p}{2}}\right| E \leq \sup _{|\xi| \geq \xi_{\max }} \frac{2}{|\xi|^{\alpha+p}} E \leq \frac{2}{\left|\xi_{\max }\right|^{p+\alpha}} E .
\end{aligned}
$$

So

$$
\left\|\left(1-\chi_{\max }(\xi)\right) \hat{g}(\xi)\right\| \leq \frac{2}{\left|\xi_{\max }\right|^{p+\alpha}} E .
$$

On the other hand, using the triangle inequality, (1.4) and (2.3), we obtain

$$
\begin{aligned}
\left\|\left(1-\chi_{\max }(\xi)\right) \hat{g}(\xi)\right\| & =\left\|\left(1-\chi_{\max }(\xi)\right)\left(\hat{g}(\xi)-\hat{g}_{\delta}(\xi)+\hat{g}_{\delta}(\xi)\right)\right\| \\
& =\left\|\left(1-\chi_{\max }(\xi)\right) \hat{g}_{\delta}(\xi)+\left(1-\chi_{\max }(\xi)\right)\left(\hat{g}(\xi)-\hat{g}_{\delta}(\xi)\right)\right\| \\
& \geq\left\|\left(1-\chi_{\max }(\xi)\right) \hat{g}_{\delta}(\xi)\right\|-\left\|\left(1-\chi_{\max }(\xi)\right)\left(\hat{g}(\xi)-\hat{g}_{\delta}(\xi)\right)\right\| \\
& \geq \tau \delta-\delta=(\tau-1) \delta .
\end{aligned}
$$

So

$$
\left\|\left(1-\chi_{\max }(\xi)\right) \hat{g}(\xi)\right\| \geq(\tau-1) \delta .
$$

Combining (2.8) with (2.9), we obtain

$$
(\tau-1) \delta \leq\left\|\left(1-\chi_{\max }(\xi)\right) \hat{g}(\xi)\right\| \leq \frac{2}{\left|\xi_{\max }\right|^{p+\alpha}} E .
$$

So

$$
\left|\xi_{\max }\right| \leq\left(\frac{2 E}{(\tau-1) \delta}\right)^{\frac{1}{p+\alpha}}
$$

Lemma 2.6 If $\xi_{\max }$ is the solution of Eq. (2.3), then the following inequality also holds:

$$
\left\|\left(1-\chi_{\max }(\xi)\right) \hat{g}(\xi)\right\| \leq(\tau+1) \delta
$$

Proof Due to (1.4) and (2.3), we obtain

$$
\begin{aligned}
\left\|\left(1-\chi_{\max }(\xi)\right) \hat{g}(\xi)\right\| & =\left\|\left(1-\chi_{\max }(\xi)\right)\left(\hat{g}(\xi)-\hat{g}_{\delta}(\xi)+\hat{g}_{\delta}(\xi)\right)\right\| \\
& \leq\left\|\left(1-\chi_{\max }(\xi)\right)\left(\hat{g}(\xi)-\hat{g}_{\delta}(\xi)\right)\right\|+\left\|\left(1-\chi_{\max }(\xi)\right) \hat{g}_{\delta}(\xi)\right\| \\
& \leq \delta+\tau \delta=(\tau+1) \delta .
\end{aligned}
$$


Now we give the main result of this section.

Theorem 2.7 Suppose that conditions (1.4) and (1.8) hold and take the solution $\xi_{\max }$ of Eq.

(2.3) as the regularization parameter, then we have the following error estimate:

$$
\begin{aligned}
\left\|f(\cdot)-f_{\delta, \xi_{\max }}(\cdot)\right\| \leq & \left((2(\tau+1))^{\frac{p}{p+\alpha}}+2\left(\frac{2}{\tau-1}\right)^{\frac{\alpha}{p+\alpha}}\right) \\
& \times E^{\frac{\alpha}{p+\alpha}} \delta^{\frac{p}{p+\alpha}}(1+o(1)) \text { as } \delta \rightarrow 0 .
\end{aligned}
$$

Proof Using the Parseval formula and the triangle inequality, we obtain

$$
\begin{aligned}
& \left\|f(\cdot)-f_{\delta, \xi_{\max }}(\cdot)\right\|=\left\|\hat{f}(\cdot)-\hat{f}_{\delta, \xi_{\max }}(\cdot)\right\| \\
& =\left\|\frac{\psi_{\alpha}^{\theta}(\xi)}{1-e^{-\psi_{\alpha}^{\theta}(\xi)}} \hat{g}(\xi)-\frac{\psi_{\alpha}^{\theta}(\xi)}{1-e^{-\psi_{\alpha}^{\theta}(\xi)}} \hat{g}_{\delta}(\xi) \chi_{\max }\right\| \\
& \leq\left\|\frac{\psi_{\alpha}^{\theta}(\xi)}{1-e^{-\psi_{\alpha}^{\theta}(\xi)}} \hat{g}(\xi)-\frac{\psi_{\alpha}^{\theta}(\xi)}{1-e^{-\psi_{\alpha}^{\theta}(\xi)}} \hat{g}(\xi) \chi_{\max }\right\| \\
& +\left\|\frac{\psi_{\alpha}^{\theta}(\xi)}{1-e^{-\psi_{\alpha}^{\theta}(\xi)}} \hat{g}(\xi) \chi_{\max }-\frac{\psi_{\alpha}^{\theta}(\xi)}{1-e^{-\psi_{\alpha}^{\theta}(\xi)}} \hat{g}_{\delta}(\xi) \chi_{\max }\right\| \\
& =\left(\int_{|\xi|>\xi_{\max }}\left|\frac{\psi_{\alpha}^{\theta}(\xi)}{1-e^{-\psi_{\alpha}^{\theta}(\xi)}}\right|^{2}|\hat{g}(\xi)|^{2} d \xi\right)^{\frac{1}{2}} \\
& +\left(\int_{|\xi| \leq \xi \max }\left(\frac{\psi_{\alpha}^{\theta}(\xi)}{1-e^{-\psi_{\alpha}^{\theta}(\xi)}}\left(\hat{g}(\xi)-\hat{g}_{\delta}(\xi)\right)\right)^{2} d \xi\right)^{\frac{1}{2}} \\
& =I_{1}+I_{2} \text {. }
\end{aligned}
$$

Using the Hölder inequality and (2.12), we obtain

$$
\begin{aligned}
I_{1}^{2}= & \int_{|\xi|>\xi \max }\left|\frac{\psi_{\alpha}^{\theta}(\xi)}{1-e^{-\psi_{\alpha}^{\theta}(\xi)}}\right|^{2}|\hat{g}(\xi)|^{2} d \xi \\
= & \int_{|\xi|>\xi \max }\left|\frac{\psi_{\alpha}^{\theta}(\xi)}{1-e^{-\psi_{\alpha}^{\theta}(\xi)}}\right|^{2}|\hat{g}(\xi)|^{\frac{2 \alpha}{p+\alpha}}|\hat{g}(\xi)|^{2\left(1-\frac{\alpha}{p+\alpha}\right)} d \xi \\
\leq & \left(\int_{|\xi|>\xi \max }\left(\left|\frac{\psi_{\alpha}^{\theta}(\xi)}{1-e^{-\psi_{\alpha}^{\theta}(\xi)}}\right|^{2}|\hat{g}(\xi)|^{\frac{2 \alpha}{p+\alpha}}\right)^{\frac{p+\alpha}{\alpha}} d \xi\right)^{\frac{\alpha}{p+\alpha}} \\
& \times\left(\int_{|\xi|>\xi \max }\left(|\hat{g}(\xi)|^{2\left(1-\frac{\alpha}{p+\alpha}\right)}\right)^{\frac{p+\alpha}{p}} d \xi\right)^{\frac{p}{p+\alpha}} \\
= & \left(\int_{|\xi|>\xi \max }\left|\frac{\psi_{\alpha}^{\theta}(\xi)}{1-e^{-\psi_{\alpha}^{\theta}(\xi)}}\right|^{\frac{2(p+\alpha)}{\alpha}}|\hat{g}(\xi)|^{2} d \xi\right)^{\frac{\alpha}{p+\alpha}}\left(\int_{\mid \xi \xi>\xi \max }|\hat{g}(\xi)|^{2} d \xi\right)^{\frac{p}{p+\alpha}} \\
= & \left(\int_{|\xi|>\xi \max }\left|\frac{\psi_{\alpha}^{\theta}(\xi)}{1-e^{-\psi_{\alpha}^{\theta}(\xi)}}\right|^{\frac{2 p}{\alpha}}\left|\frac{\psi_{\alpha}^{\theta}(\xi)}{1-e^{-\psi_{\alpha}^{\theta}(\xi)}} \hat{g}(\xi)\right|^{2} d \xi\right)^{\frac{\alpha}{p+\alpha}}\left(\int_{|\xi|>\xi \max }|\hat{g}(\xi)|^{2} d \xi\right)^{\frac{p}{p+\alpha}} \\
= & \left(\int_{\mid \xi \xi>\xi \max }\left(1+\xi^{2}\right)^{-p}\left|\frac{\psi_{\alpha}^{\theta}(\xi)}{1-e^{-\psi_{\alpha}(\xi)}}\right|^{\frac{2 p}{\alpha}}\left(1+\xi^{2}\right)^{p}|\hat{f}(\xi)|^{2} d \xi\right)^{\frac{\alpha}{p+\alpha}} \\
& \times\left(\int_{|\xi|>\xi \max }|\hat{g}(\xi)|^{2} d \xi\right)^{\frac{p}{p+\alpha}}
\end{aligned}
$$




$$
\begin{aligned}
& \leq\left.\left.\sup _{|\xi|>\xi \max }\left|\left(1+\xi^{2}\right)^{-p}\right| \frac{|\xi|^{\alpha}}{1-e^{-|\xi|^{\alpha} \cos \left(\frac{\theta \pi}{2}\right)}}\right|^{\frac{2 p}{\alpha}}\right|^{\frac{\alpha}{p+\alpha}} E^{\frac{2 \alpha}{p+\alpha}}\left(\int_{|\xi|>\xi \max }|\hat{g}(\xi)|^{2} d \xi\right)^{\frac{p}{p+\alpha}} \\
& \leq \sup _{|\xi|>\xi \max }\left|\frac{1}{1-e^{-|\xi|^{\alpha} \cos \left(\frac{\theta \pi}{2}\right)}}\right|^{\frac{2 p}{p+\alpha}} E^{\frac{2 \alpha}{p+\alpha}}\left\|\left(1-\chi_{\max }\right)|\hat{g}(\xi)|\right\|^{\frac{2 p}{p+\alpha}} \\
& \leq 2^{\frac{2 p}{p+\alpha}} E^{\frac{2 \alpha}{p+\alpha}}((\tau+1) \delta)^{\frac{2 p}{p+\alpha}} .
\end{aligned}
$$

So

$$
I_{1} \leq 2^{\frac{p}{p+\alpha}} E^{\frac{\alpha}{p+\alpha}}((\tau+1) \delta)^{\frac{p}{p+\alpha}}=2^{\frac{p}{p+\alpha}}(\tau+1)^{\frac{p}{p+\alpha}} E^{\frac{\alpha}{p+\alpha}} \delta^{\frac{p}{p+\alpha}} .
$$

Combining (1.4) with (2.4), we obtain

$$
\begin{aligned}
I_{2}= & \left(\int_{|\xi| \leq \xi \max }\left(\frac{(i \xi)^{\alpha}}{1-e^{-|\xi|^{\alpha} \cos \left(\frac{\theta \pi}{2}\right)}}\left(\hat{g}(\xi)-\hat{g}_{\delta}(\xi)\right)\right)^{2} d \xi\right)^{\frac{1}{2}} \leq \sup _{|\xi| \leq \xi \max }\left|\frac{|\xi|^{\alpha}}{1-e^{-|\xi|^{\alpha} \cos \left(\frac{\theta \pi}{2}\right)}}\right| \delta \\
& \leq \sup _{|\xi| \leq 1}\left|\frac{|\xi|^{\alpha}}{1-e^{-|\xi|^{\alpha} \cos \left(\frac{\theta \pi}{2}\right)}}\right| \delta+\sup _{1<|\xi| \leq \xi \max }\left|\frac{|\xi|^{\alpha}}{1-e^{-|\xi|^{\alpha} \cos \left(\frac{\theta \pi}{2}\right)}}\right| \delta \\
& \leq\left(\frac{2}{\cos \left(\frac{\theta \pi}{2}\right)}+2 \xi_{\max }^{\alpha}\right) \delta .
\end{aligned}
$$

Using (2.7), we obtain

$$
\begin{aligned}
I_{2} & \leq\left(\frac{2}{\cos \left(\frac{\theta \pi}{2}\right)}+2 \xi_{\max }^{\alpha}\right) \delta \leq 2\left(\frac{2 E}{(\tau-1) \delta}\right)^{\frac{\alpha}{p+\alpha}} \delta+\frac{2}{\cos \left(\frac{\theta \pi}{2}\right)} \delta \\
& =2\left(\frac{2}{\tau-1}\right)^{\frac{\alpha}{p+\alpha}} E^{\frac{\alpha}{p+\alpha}} \delta^{\frac{p}{p+\alpha}}+\frac{2}{\cos \left(\frac{\theta \pi}{2}\right)} \delta .
\end{aligned}
$$

So

$$
I_{2} \leq 2\left(\frac{2}{\tau-1}\right)^{\frac{\alpha}{p+\alpha}} E^{\frac{\alpha}{p+\alpha}} \delta^{\frac{p}{p+\alpha}}+\frac{2}{\cos \left(\frac{\theta \pi}{2}\right)} \delta .
$$

Combining (2.14) with (2.15), we obtain

$$
\begin{aligned}
\left\|f(\cdot)-f_{\delta, \xi_{\max }}(\cdot)\right\| & \leq 2^{\frac{p}{p+\alpha}}(\tau+1)^{\frac{p}{p+\alpha}} E^{\frac{\alpha}{p+\alpha}} \delta^{\frac{p}{p+\alpha}}+2\left(\frac{2}{\tau-1}\right)^{\frac{\alpha}{p+\alpha}} E^{\frac{\alpha}{p+\alpha}} \delta^{\frac{p}{p+\alpha}}+\frac{2}{\cos \left(\frac{\theta \pi}{2}\right)} \delta \\
& =\left((2(\tau+1))^{\frac{p}{p+\alpha}}+2\left(\frac{2}{\tau-1}\right)^{\frac{\alpha}{p+\alpha}}\right) E^{\frac{\alpha}{p+\alpha}} \delta^{\frac{p}{p+\alpha}}(1+o(1)) \quad \text { as } \delta \rightarrow 0 .
\end{aligned}
$$

The proof of Theorem 2.7 is completed.

\section{Several numerical examples}

In this section, we present two numerical examples to verify the validity of the theoretical result of these methods. Moreover, we would like to compare numerical results of the $a$ posteriori parameter choice (2.3) with one of the a priori parameter choice rules $\xi_{\max }=$ $\left(\frac{E}{\delta}\right)^{\frac{1}{p+\alpha}}$ in [27]. 
The numerical examples were constructed in the following way: First we selected the exact solution $f(x)$ and obtained the exact data function $g(x)$ through solving the forward problems. Then we added a normally distributed perturbation to each data function and obtained vectors $g_{\delta}(x)$. Finally we obtained the regularization solution through solving the inverse problem. The bisection method is used to solve Eq. (2.3) with $\tau=1.1$. In the following experiments, we choose $x \in[-5,5]$.

Suppose that the sequence $\left\{g_{k}\right\}_{k=0}^{n}$ represents samples from the function $g(x)$ on an equidistant grid, then we add a random uniform perturbation to each data, which forms the vector $g_{\delta}$, i.e.,

$$
g_{\delta}=g+\varepsilon \operatorname{randn}(\operatorname{size}(g))
$$

where

$$
g=\left(g\left(x_{1}\right), \ldots, g\left(x_{n}\right)\right)^{T}, \quad x_{i}=-5+(i-1) \Delta x, \Delta x=\frac{10}{n-1}, i=1,2, \ldots, n .
$$

The function 'randn(.)' generates arrays of random numbers whose elements are normally distributed with mean 0 , variance $\sigma^{2}=1$. 'randn $(\operatorname{size}(g)$ )' returns an array of random entries that is of the same size as $g$. The total noise level $\delta$ can be measured in the sense of root mean square error (RMSE) according to

$$
\delta=\left\|g_{\delta}-g\right\|_{l^{2}}=\left(\frac{1}{n} \sum_{i=1}^{n}\left(g_{i}-g_{i, \delta}\right)^{2}\right)^{\frac{1}{2}} .
$$

The approximation of the regularization solution is computed by using the fast Fourier transform algorithm [28].

Example 1 Consider a piecewise smooth unknown source as follows:

$$
f(x)= \begin{cases}0, & -5 \leq x \leq-1 \\ 1-x^{2}, & -1<x \leq 1 \\ 0, & 1<x \leq 5\end{cases}
$$

Example 2 Consider the following discontinuous unknown source:

$$
f(x)= \begin{cases}0, & -5 \leq x \leq-\frac{5}{2} \\ 1, & -\frac{5}{2}<x \leq \frac{5}{2} \\ 0, & \frac{5}{2}<x \leq 5\end{cases}
$$

From Figures 1-8, we find that the smaller $\varepsilon$, the better the computed approximation is, and the smaller the $\alpha$ is, the better the computed approximation is. These are consistent with our theoretical analysis. Moreover, we can also easily find that the $a$ posteriori parameter choice rule also works well. Finally, from Figures 1-8, it can be seen that the numerical solutions of Example 2 are less ideal than these of Example 1. It is not difficult to see that the well-known Gibbs phenomenon and the recovered data near the discontinuities points are not accurate. 


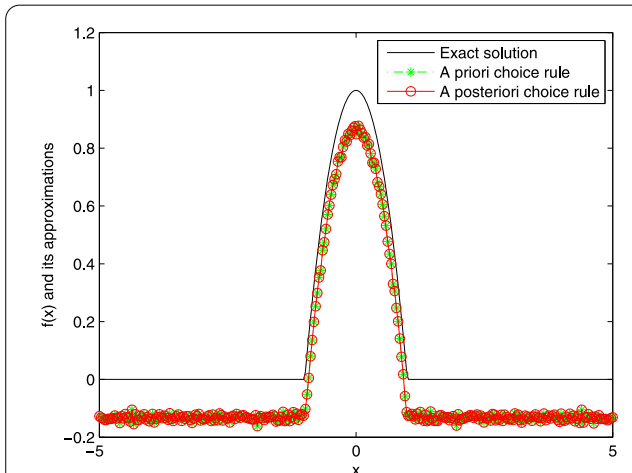

(a)

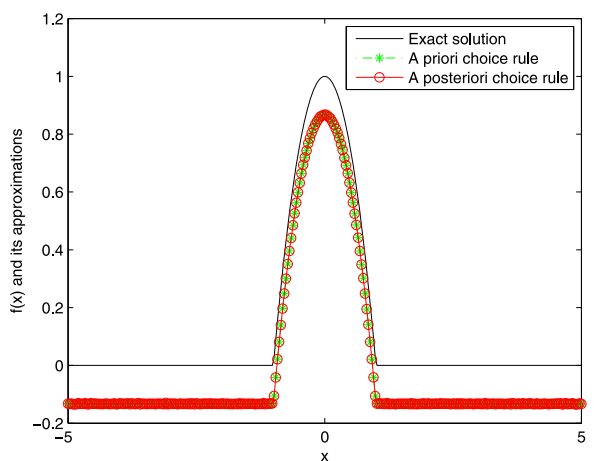

(b)

Figure 1 The comparison of the numerical effects between the exact solution and its computed approximations for $p=1, \theta=0.1$ and $\alpha=0.3$ with Example 1: (a) $\varepsilon=0.1$, (b) $\varepsilon=0.01$.

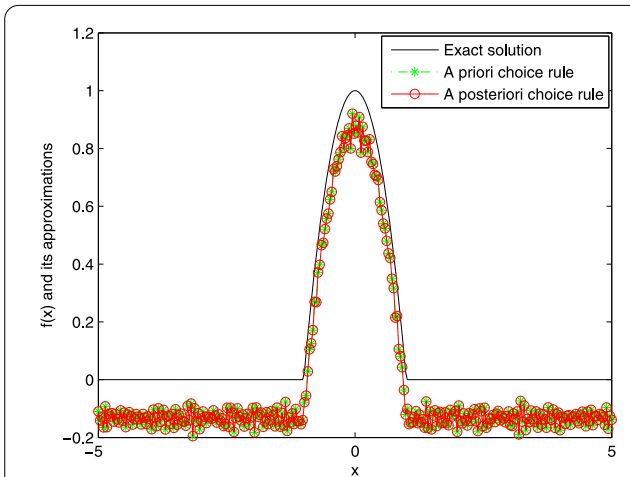

(a)

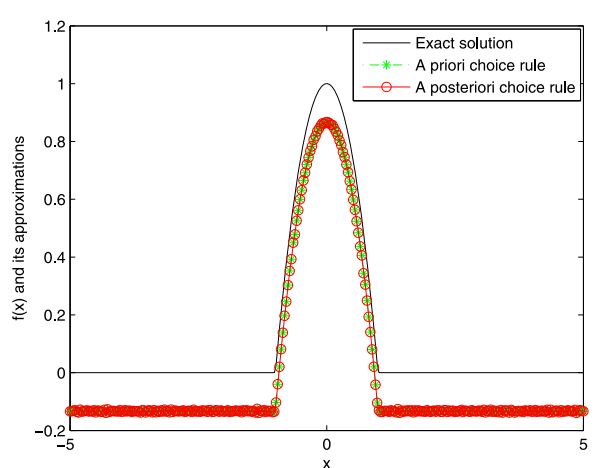

(b)

Figure 2 The comparison of the numerical effects between the exact solution and its computed approximations for $p=1, \theta=0.1$ and $\alpha=0.7$ with Example 1: (a) $\varepsilon=0.1$, (b) $\varepsilon=0.01$.

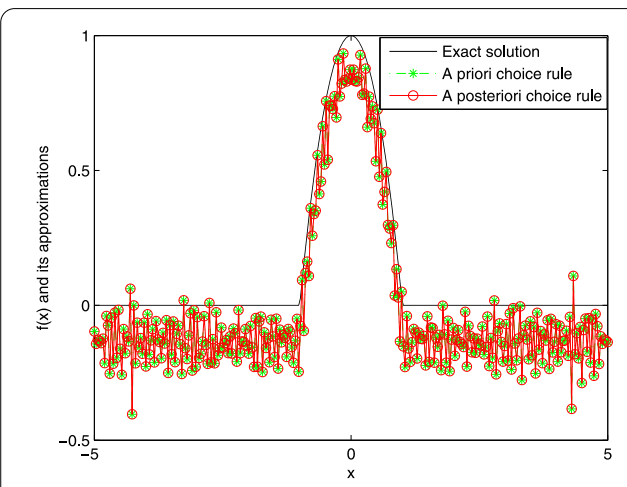

(a)

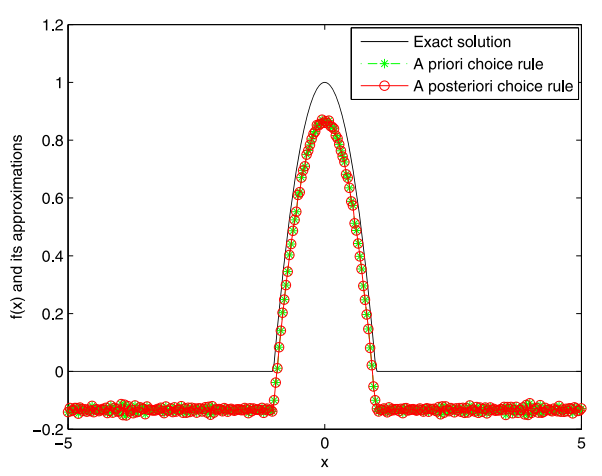

(b)

Figure 3 The comparison of the numerical effects between the exact solution and its computed approximations for $p=1, \theta=0.1$ and $\alpha=1.1$ with Example 1: (a) $\varepsilon=0.1$, (b) $\varepsilon=0.01$. 


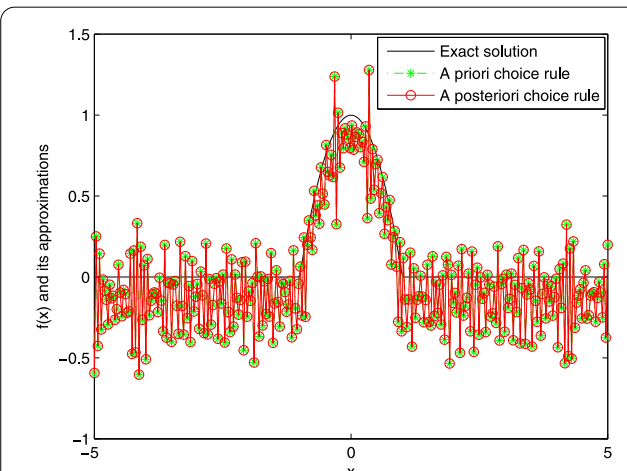

(a)

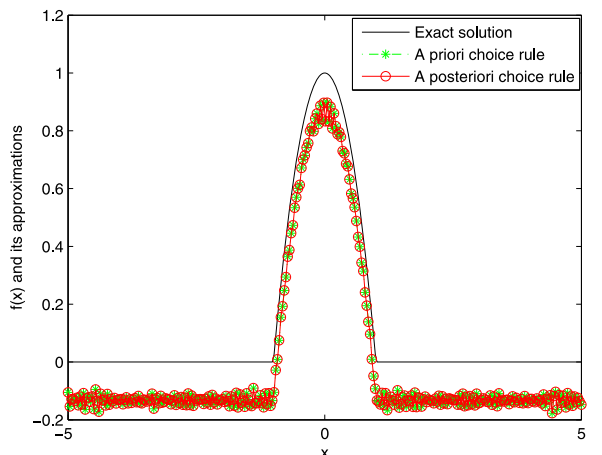

(b)

Figure 4 The comparison of the numerical effects between the exact solution and its computed approximations for $p=1, \theta=0.1$ and $\alpha=1.4$ with Example 1: (a) $\varepsilon=0.1$, (b) $\varepsilon=0.01$.

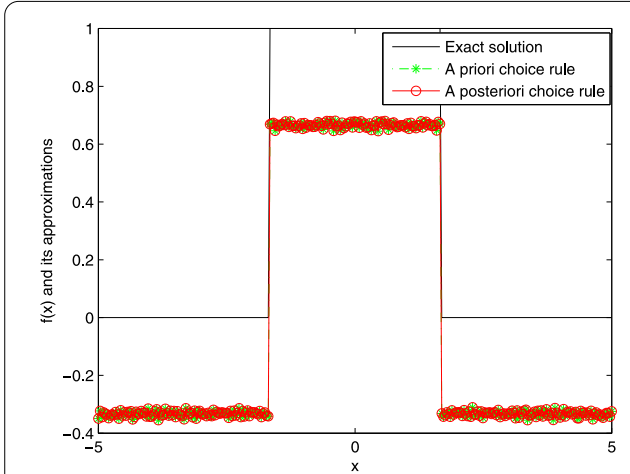

(a)

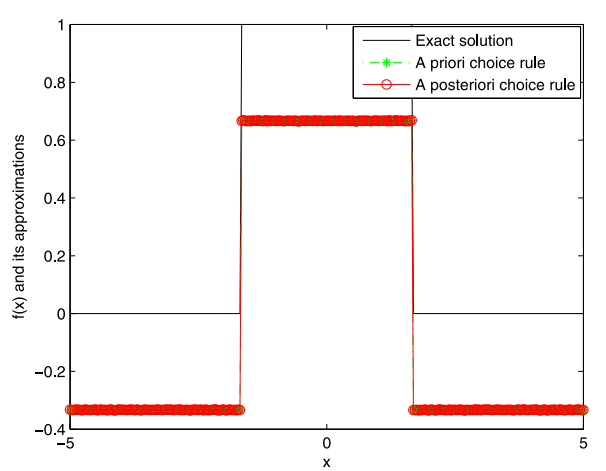

(b)

Figure 5 The comparison of the numerical effects between the exact solution and its computed approximations for $p=1, \theta=0.1$ and $\alpha=0.3$ with Example 2: (a) $\varepsilon=0.1$, (b) $\varepsilon=0.01$.

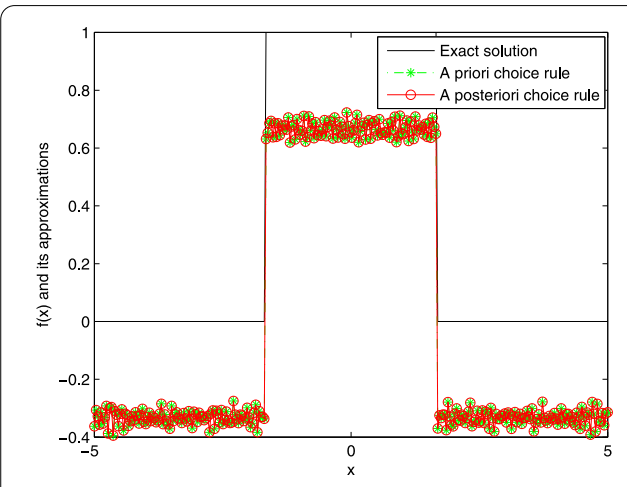

(a)

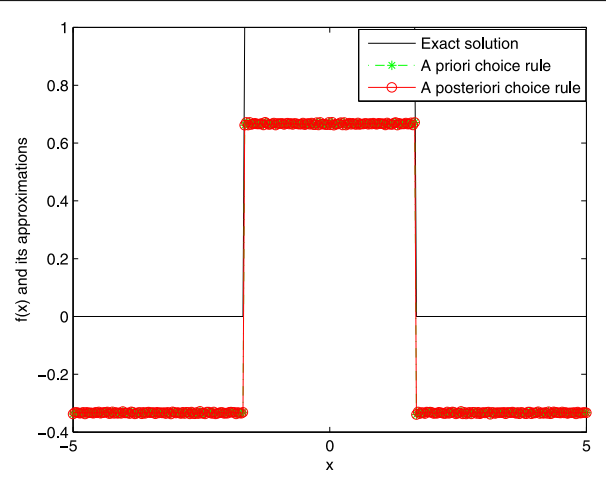

(b)

Figure 6 The comparison of the numerical effects between the exact solution and its computed approximations for $p=1, \theta=0.1$ and $\alpha=0.7$ with Example 2: (a) $\varepsilon=0.1$, (b) $\varepsilon=0.01$. 


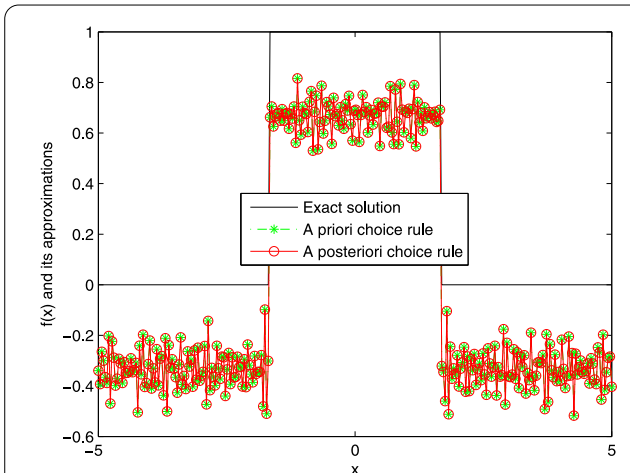

(a)

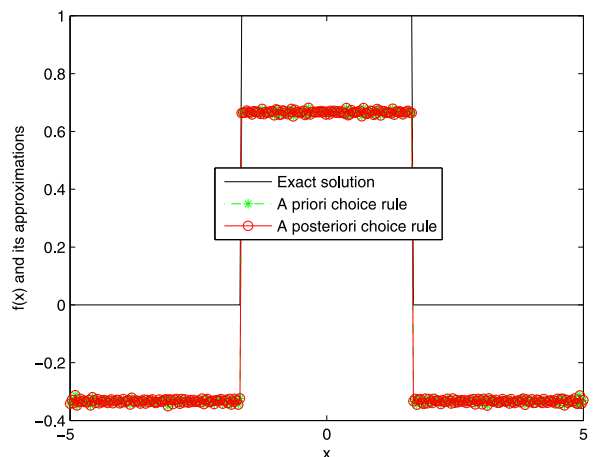

(b)

Figure 7 The comparison of the numerical effects between the exact solution and its computed approximations for $p=1, \theta=0.1$ and $\alpha=1.1$ with Example 2: (a) $\varepsilon=0.1$, (b) $\varepsilon=0.01$.

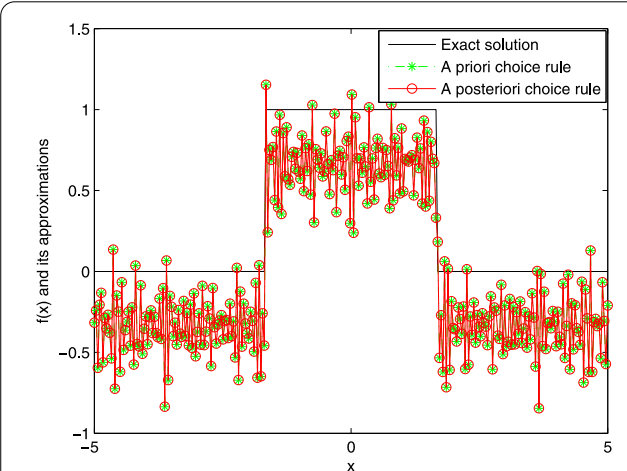

(a)

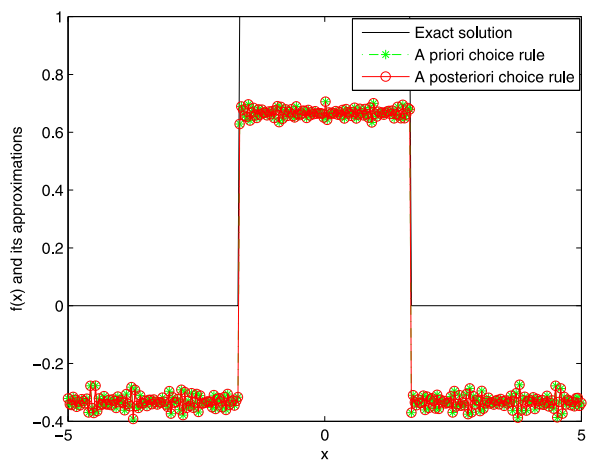

(b)

Figure 8 The comparison of the numerical effects between the exact solution and its computed approximations for $p=1, \theta=0.1$ and $\alpha=1.4$ with Example 2: (a) $\varepsilon=0.1$, (b) $\varepsilon=0.01$.

\section{Conclusions}

In this paper, the Fourier method is used to identify the unknown source term depending only on the spatial variable for a Riesz-Feller space-fractional diffusion equation. We propose to choose the regularization parameter by an a posteriori rule using the discrepancy principle. The corresponding error estimate between the exact solution and the regularization solution is obtained. Numerical tests show that the proposed scheme is accurate, stable and convergent with respect to decreasing the amount of noise added into the data.

Competing interests

The authors declare that they have no competing interests.

Authors' contributions

The main idea of this paper was proposed by FY and X-XL prepared the manuscript initially and performed all the steps of the proofs in this research. All authors read and approved the final manuscript.

\section{Acknowledgements}

The project is supported by the National Natural Science Foundation of China (No. 11171136, No. 11261032), the Distinguished Young Scholars Fund of Lan Zhou University of Technology (Q201015), the basic scientific research business expenses of Gansu province college and the Natural Science Foundation of Gansu province (1310RJYA021). 


\section{References}

1. Metzler, R, Klafter, J: The random walk's guide to anomalous diffusion: a fractional dynamics approach. Phys. Rep. 339 $1-77(2000)$

2. Metzler, R, Klafter, J: The restaurant at the end of the random walk: recent developments in the description of anomalous transport by fractional dynamics. J. Phys. A, Math. Gen. 37, R161-R208 (2004)

3. Meerschaert, MM, Tadjeran, C: Finite difference approximations for fractional advection-dispersion flow equations. J. Comput. Appl. Math. 172, 65-77 (2003)

4. Chen, MH, Deng, WH: Fourth order accurate scheme for the space fractional diffusion equations. SIAM J. Numer. Anal. 52(3), 1418-1438 (2014)

5. Chen, MH, Deng, WH: Fourth order difference approximations for space Riemann-Liouville derivatives based on weighted and shifted Lubich difference operators. Commun. Comput. Phys. 16, 516-540 (2014)

6. Chen, MH, Deng, WH, Wu, YJ: Superlinearly convergent algorithms for the two-dimensional space-time Caputo-Riesz fractional diffusion equation. Appl. Numer. Math. 70, 22-41 (2013)

7. Ervin, VJ, Heuer, N, Roop, JP: Numerical approximation of a time dependent, nonlinear, space-fractional diffusion equation. SIAM J. Numer. Anal. 45, 572-591 (2007)

8. Liu, F, Anh, V, Turner, I: Numerical solution of the space fractional Fokker-Planck equation. J. Comput. Appl. Math. 166 209-219 (2004)

9. Meerschaert, MM, Tadjeran, C: Finite difference approximations for two-sided space-fractional partial differential equations. Appl. Numer. Math. 56, 80-90 (2006)

10. Ahmadabadi, MN, Arab, M, Malek Ghaini, FM: The method of fundamental solutions for the inverse space-dependent heat source problem. Eng. Anal. Bound. Elem. 33, 1231-1235 (2009)

11. Cannon, JR, Duchateau, P: Structural identification of an unknown source term in a heat equation. Inverse Probl. 14 535-551 (1998)

12. Cheng, W, Fu, CL: Identifying an unknown source term in a spherically symmetric parabolic equation. Appl. Math. Lett. 26, 387-391 (2013)

13. Farcas, A, Lesnic, D: The boundary-element method for the determination of a heat source dependent on one variable. J. Eng. Math. 54, 375-388 (2006)

14. Li, GS: Data compatibility and conditional stability for an inverse source problem in the heat equation. Appl. Math Comput. 173, 566-581 (2006)

15. Liu, FB: A modified genetic algorithm for solving the inverse heat transfer problem of estimating plan heat source. Int. J. Heat Mass Transf. 51, 3745-3752 (2008)

16. Liu, CH: A two-stage LGSM to identify time-dependent heat source through an internal measurement of temperature. Int. J. Heat Mass Transf. 52, 1635-1642 (2009)

17. $\mathrm{Ma}, \mathrm{YJ}, \mathrm{Fu}, \mathrm{CL}$, Zhang, YX: Identification of an unknown source depending on both time and space variables by a variational method. Appl. Math. Model. 36, 5080-5090 (2012)

18. Zhang, $Y, X u, X$ : Inverse source problem for a fractional diffusion equation. Inverse Probl. 27, 035010 (2011)

19. Wei, H, Chen, W, Sun, HG, Li, XC: A coupled method for inverse source problem of spatial fractional anomalous diffusion equations. Inverse Probl. Sci. Eng. 18, 945-956 (2010)

20. Yang, F, Fu, CL: A mollification regularization method for the inverse spatial-dependent heat source problem. J. Comput. Appl. Math. 255, 555-567 (2014)

21. Wei, T, Zhang, ZQ: Reconstruction of a time-dependent source term in a time-fractional diffusion equation. Eng. Anal. Bound. Elem. 37, 23-31 (2013)

22. Wang, JG, Zhou, YB, Wei, T: Two regularization methods to identify a space-dependent source for the time-fractional diffusion equation. Appl. Numer. Math. 68, 39-57 (2013)

23. Zhang, ZQ, Wei, T: Identifying an unknown source in time-fractional diffusion equation by a truncation method. Appl. Math. Comput. 219, 5972-5983 (2013)

24. Mainardi, F, Luchko, Y, Pagnini, G: The fundamental solution of the space-time fractional diffusion equation. Fract. Calc. Appl. Anal. 4, 153-192 (2001)

25. Benson, DA, Wheatcraft, SW, Meerschaert, MM: The fractional-order governing equation of Lévy motion. Water Resour. Res. 36, 1413-1423 (2000)

26. Gorenflo, R, Mainardi, F, Moretti, D, Pagnini, G, Paradisi, P: Discrete random walk models for space-time fractional diffusion. Chem. Phys. 284, 521-541 (2002)

27. Tian, WY, Li, C, Deng, WH, Wu, YJ: Regularization methods for unknown source in space fractional diffusion equation. Math. Comput. Simul. 85, 45-56 (2012)

28. Eldén, L, Berntsson, F, Regińska, T: Wavelet and Fourier methods for solving the sideways heat equation. SIAM J. Sci. Comput. 21, 2187-2205 (2000)

29. Xiong, XT, Fu, CL, Li, HF: Fourier regularization method of a sideways heat equation for determining surface heat flux. J. Math. Anal. Appl. 317, 331-348 (2006)

30. Fu, CL, Xiong, XT, Qian, Z: Fourier regularization for a backward heat equation. J. Math. Anal. Appl. 331, 472-480 (2007)

31. Qian, Z, Fu, CL, Xiong, XT, Wei, T: Fourier truncation method for high order numerical derivatives. Appl. Math. Comput. 181, 940-948 (2006)

32. Regińska, T, Regiński, K: Approximate solution of a Cauchy problem for the Helmholtz equation. Inverse Probl. 22 , 975-989 (2006)

33. Dou, FF, Fu, CL, Yang, FL: Optimal error bound and Fourier regularization for identifying an unknown source in the heat equation. J. Comput. Appl. Math. 230, 728-737 (2008)

34. Yang, F, Fu, CL: Two regularization methods to identify time-dependent heat source through an internal measurement of temperature. Math. Comput. Model. 53, 793-804 (2011)

\subsection{6/1029-242X-2014-434}

Cite this article as: Li et al.: An a posteriori Fourier regularization method for identifying the unknown source of the space-fractional diffusion equation. Journal of Inequalities and Applications 2014, 2014:434 